\title{
Epidermal barrier formation and recovery in skin disorders
}

\author{
Julia A. Segre \\ Genetics and Molecular Biology Branch, National Human Genome Research Institute, National Institutes of Health, Bethesda, Maryland, USA.
}

\begin{abstract}
Skin is at the interface between the complex physiology of the body and the external, often hostile, environment, and the semipermeable epidermal barrier prevents both the escape of moisture and the entry of infectious or toxic substances. Newborns with rare congenital barrier defects underscore the skin's essential role in a terrestrial environment and demonstrate the compensatory responses evoked ex utero to reestablish a barrier. Common inflammatory skin disorders such as atopic dermatitis and psoriasis exhibit decreased barrier function, and recent studies suggest that the complex response of epidermal cells to barrier disruption may aggravate, maintain, or even initiate such conditions. Either aiding barrier reestablishment or dampening the epidermal stress response may improve the treatment of these disorders. This Review discusses the molecular regulation of the epidermal barrier as well as causes and potential treatments for defects of barrier formation and proposes that medical management of barrier disruption may positively affect the course of common skin disorders.
\end{abstract}

\section{Molecular regulation of barrier acquisition and prematurity}

Mammalian epidermis is a stratified epithelium that retains the ability to self renew under both homeostatic and injury conditions by maintaining a population of mitotically active cells in the hair follicles and innermost basal layer (Figure 1) (1-3). The major barrier resides within the exterior layers of the epidermis, which are sloughed off and repopulated from these inner cells. The process of terminal differentiation begins when basal cells concomitantly withdraw from the cell cycle and lose their ability to adhere to the basement membrane. In the intermediate spinous layers, the cells reinforce a durable cytoskeletal framework of keratin filaments to provide the mechanical strength necessary to resist physical trauma. In the granular layers, lipids are produced inside lamellar bodies, keratins are bundled into macrofibrils through their association with filaggrin, and a cornified envelope (CE) is assembled by sequential incorporation of precursor proteins directly underneath the plasma membrane. As the cell membrane disintegrates, the subsequent calcium influx activates the transglutaminase (TGM) enzyme to irreversibly cross-link the CE proteins, creating a tough, insoluble sac that surrounds the keratin fibers. Finally, lipids are extruded into the intercellular space onto the CE scaffold. This barrier, once built, is analogous to bricks and mortar, with the keratin macrofibrils and CEs forming the bricks and the extruded lipids forming the mortar to seal together the CEs (4-6). Recent experimental results have also demonstrated an essential role for tight junctions, located in the granular layer, in forming the epidermal barrier (7). This process of differentiation from a mitotically active basal cell to a squame, a terminally differentiated squamous cell, is maintained throughout life as part of epidermal regeneration $(8,9)$.

During development, the fetus requires a barrier to prevent intrauterine infection and to serve as the interface with the amniotic fluid. Shortly after gastrulation, the embryonic ectoderm gives rise

Nonstandard abbreviations used: $\mathrm{AD}$, atopic dermatitis; $\mathrm{CE}$, cornified envelope; Klf4, Kruppel-like factor 4; SPINK5, serine protease inhibitor, Kazal type 5; SPRR, small proline-rich protein; TGM, transglutaminase; Tslp, thymic stromal lymphopoietin. Conflict of interest: The author has declared that no conflict of interest exists. Citation for this article: J. Clin. Invest. 116:1150-1158 (2006). doi:10.1172/JCI28521. to the presumptive epidermis, and by approximately 8 weeks of gestation, basal cells cover the body surface. For the next 5 months, the periderm provides a temporary barrier suitable for the aqueous environment in utero. The periderm is derived from basal keratinocytes but differs from differentiated adult squames in the cell morphology, keratin profile, molecular architecture of the tight junctions, and presence of microvilli on the apical surface extending into the amniotic fluid (10). Beneath the periderm, the epidermis stratifies and differentiates so that by week 34 of gestation the skin is competent to serve as a barrier in a terrestrial environment. At this point, the periderm cells are sloughed to form part of the vernix caseosa that covers the newborn. During the second and third trimesters of pregnancy, the volume of the fetus grows at a rapid rate, which requires an even larger increase of the fetus' surface area. Acquisition of a barrier that is competent to survive in the terrestrial environment requires cross-linking of the CEs and constrains increases in the surface area of the embryo. Therefore, it is not advantageous to develop the terrestrial competent barrier too early in development. However, the barrier must still be acquired prior to the typical end of gestation at 40 weeks in case a premature birth occurs. Incomplete barrier acquisition manifests in premature infants as dehydration, electrolyte imbalance, and poor thermoregulation and predisposes to infection. Although the transition to the terrestrial environment ex utero accelerates the epidermal differentiation program, an infant born at 25-30 weeks requires 2-4 weeks to develop a fully functional barrier (11).

Two animal models have demonstrated how this process of barrier acquisition can be accelerated in utero. First, corticosteroid injections, acting through the glucocorticoid receptor, have been demonstrated to accelerate barrier acquisition $(12,13)$. Conversely, genetically altered mice with a corticosteroid deficiency have a developmental delay of barrier maturation, with defects in both lipid and structural protein synthesis (14). This animal model is particularly relevant since antenatal corticosteroids are standardof-care treatment to accelerate lung surfactant maturation when a premature birth is anticipated. Second, expression of the transcription factor Kruppel-like factor 4 (Klf4) is necessary to establish the prenatal epidermal barrier in mice (15). As well, ectopic expression of Klf4 accelerates barrier acquisition within a field of 


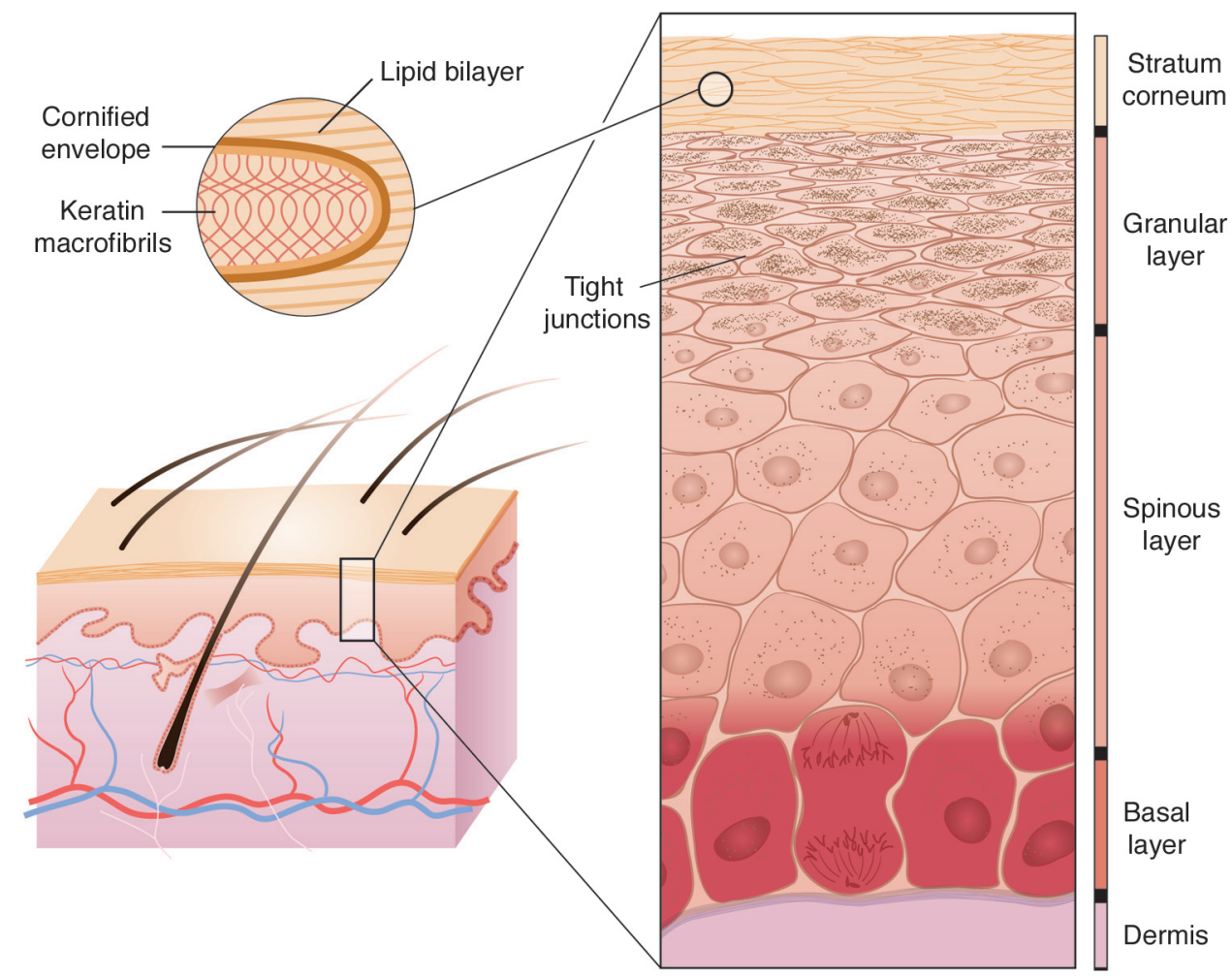

\section{Figure 1}

Schematic diagram of the stages of epidermal differentiation, resulting in a permeability barrier. Epidermal keratinocytes undergo a linear program of differentiation from mitotically active basal cells to transcriptionally active spinous cells to enucleated granular cells, resulting finally in differentiated squames in the stratum corneum. As shown in the inset, squames, which provide the primary barrier, are composed of keratin macrofibrils and crosslinked cornified envelopes encased in lipid bilayers. Tight junctions, located in the granular layer, also play an essential role in retaining the water content of the body. competence established by the expression of the late CE proteins $(16,17)$. Intriguingly, earlier studies showed a cooperative synergistic activation of a test promoter by glucocorticoid receptor and Klf transcription factors, suggesting that these factors may act to coordinately regulate downstream targets. Ex utero, corticosteroids are not given to premature infants because of adverse side effects, including immune suppression. However, some of the genes regulated by KLF4 and corticosteroids may be suitable pharmaceutical targets. Hopefully, by understanding how barrier acquisition is programmed in utero, we can expedite the process ex utero.

\section{Human congenital barrier deficiencies and animal models}

The genetically and clinically diverse epidermal differentiation or cornification disorders are classified as ichthyoses, derived from the Greek root ichthys, meaning "fish," and referring to the scaly presentation of the skin. The scaly appearance is the gross morphological presentation of hyperkeratosis, thickening of the stratum corneum, which results from either an increase in cell proliferation, a delay in cell shedding, or both. Although ichthyosis is a more general classification, this perturbation in the balance of epidermal proliferation and differentiation does typically result in barrier deficiency. Ichthyoses can differ by age of onset, type and location of scale, erythroderma, and associated abnormalities of associated skin appendages. Ichthyosis is often the first component of a multisystem disorder to be diagnosed. Classification of ichthyoses has been greatly aided by the discovery of the specific genetic defect that underlies many of these disorders. The immediate translational benefit of this research is the development of prenatal and perinatal genetic screening to aid in disease diagnosis and to predict the prognosis or associated outcomes. Identification of the mutated gene underlying the ichthyosis is particularly beneficial for multisystem disorders in which the skin manifestation is first to be diagnosed.

The discovery that the defects that underlie many of the severe congenital ichthyoses are in genes encoding proteins that form or regulate synthesis of lipids and CEs underscores the importance of orderly differentiation in barrier acquisition (18). As a comprehensive review of the molecular genetics of the ichthyoses was recently published (18), here only a few classical and recent discoveries to illustrate key points will be described. Lamellar ichthyosis newborns, with an incidence of 1 in 300,000, present encased in a translucent, taut collodion membrane. Desquamation of this membrane occurs over the first 2 weeks of life, and the body surface is covered by large, plate-like scales that continue to exhibit increased transepidermal water loss. Linkage to multiple regions of the genome suggests that a diversity of genetic defects underlies lamellar ichthyosis. As the specific genetic defects are identified, the encoded proteins now appear to assemble into 2 discrete pathways that govern either $\mathrm{CE}$ or lipid function in the stratum corneum. Loss of the TGM1 enzyme that cross-links the CEs results in lamellar ichthyosis $(19,20)$. Alternatively, lamellar ichthyosis can be caused by aberrant lipid extrusion due to decreased function of the epidermal lipid transporter ATP-binding cassette, subfamily A, type 12 (ABCA12) or decreased lipoxygenase activity due to mutations in the cytochrome P450 gene CYP4F22 $(21,22)$. Interestingly, mutations in other proteins involved in this metabolic pathway converting arachidonic acid to compounds of the 12-lipoxygenase family, including ALOX12B, ALOXE3, and Ichthyin, underlie skin disorders with presentations that sometimes overlap lamellar ichthyosis (23-25). Complete loss of ABCA12 function results in an even more severe congenital disorder known as harlequin ichthyosis $(26,27)$. Defects in serine protease inhibitor, Kazal type 5 (SPINK5) underlie the rare Netherton syndrome, which is charac- 
terized by a severe congenital barrier defect, resolving into ichthyosis linearis circumflexa with associated hair shaft abnormalities and atopic dermatitis (AD) (28).

As these infants with congenital barrier deficiencies transition ex utero the degree, type, and location of scale can vary depending on the underlying genetic defect. One remarkable exception to the continuation of the barrier deficiency observed at birth is the "selfhealing collodion babies," which have an amino acid change in the TGM1 protein at a site that chelates water only at the higher hydrostatic pressure in utero. Molecular models and biochemical assays predict that, in the drier ex utero environment, the mutant protein attains a different conformation and possesses approximately $80 \%$ of normal TGM1 activity (29). These infants are a unique example of how specific protein mutations may have a more or less severe effect in the distinct in utero and ex utero environments.

Ichthyosis vulgaris, which is a mild but extremely common form of ichthyosis, is a complementary example of a skin disorder that typically does not affect newborns, but rather manifests in the first year of life as fine white scales. Very recently, mutations in the gene encoding the filaggrin protein, which bundles the keratin filaments in CE formation, were determined to underlie ichthyosis vulgaris (30). Two mutations that would both result in a complete loss of the cleaved filaggrin product were identified in 15 different families. Ichthyosis vulgaris exhibits a semidominant mode of inheritance, with homozygous and heterozygous mutations in the filaggrin gene underlying the severely (prominent scale) and moderately affected (mild scale) forms, respectively. Population screening revealed that approximately $7 \%$ of European controls are heterozygous for a mutation in the filaggrin gene, making this one of the most common human single-gene disorders. Although the incidence of ichthyosis vulgaris is estimated at 1 in 250, these studies suggest that heterozygous null mutations in filaggrin may not always be symptomatic, due to incomplete penetrance, seasonal variation, and a subtle phenotype. However, these mutations may still result in a minor breach of barrier that may predispose these individuals to other disorders, as will be discussed below. Finally, it is intriguing to speculate whether an evolutionary advantage is derived from an impaired barrier, which fixed these filaggrin mutations in certain populations.

The different manifestations of barrier disorders in utero and ex utero drives us to ask the question: are these clinical presentations a direct result of distinct programs of epidermal differentiation? Alternatively, a collodion membrane may provide the most protection in utero against the sterile aqueous environment, whereas the hyperkeratotic scaly stratum corneum may be the best protection against the ex utero terrestrial environment. Both types of aberrant stratum corneum may be the product of compensatory reactive pathways designed to minimize the inherent barrier deficiency. In summary, it is important to consider that the clinical presentation of a barrier defect may represent a combination of the primary defect and the skin's compensatory response to restore the barrier.

Gene targeting in mice has created animal models in which to study both the primary defect and possible compensatory responses ex utero observed in patients with congenital barrier defects. These animals have also contributed to the identification of additional pathways involved in barrier acquisition. As one example, mice with a targeted ablation of the $\operatorname{Tg} m 1$ gene, encoding the enzyme that cross-links the $\mathrm{CE}$, are born with severe epidermal barrier defects (31). Unlike the patients with lamellar ichthyosis, these mice do not survive the perinatal period, which may reflect either the larger surface area-to-volume ratio of mice compared with humans or the lack of sufficient intensive care for newborn mice. To study the postnatal development of skin in Tgm1-deficient mice, investigators grafted skin from newborn $\mathrm{Tgm1^{-/- }}$ mice onto immunocompromised mice (32). Within 2 weeks, this grafted $\mathrm{Tgm}^{1^{-1}}$ murine skin developed scaly, plate-like structures with a normal rate of transepidermal water loss. When the scales were removed, transepidermal water loss rose to a level as high as that observed in $\operatorname{Tgm}^{-/-}$neonates. This suggests that development of plate-like scales atop the skin of $\operatorname{Tgm}^{-/-}$mice represents a compensatory mechanism that prevents transepidermal water loss in the absence of cross-linked CEs. These murine experiments also suggest that the scaly, plate-like structures that cover the body of a patient with lamellar ichthyosis may be beneficial and perhaps should not be removed with retinoid treatment or soaking.

As a second unique example of a compensatory response, the protein loricrin comprises $80 \%$ of the protein content of the $\mathrm{CE}$, and yet when the gene encoding loricrin is deleted from the genome, the newborn mice exhibit a normal barrier at birth (33). Loricrin-deficient embryos exhibit a temporal delay in embryonic barrier competence until upregulation of the expression of other components of the CE compensate. Loricrin-deficient CEs are more fragile, suggesting that these mice might not respond as well as their wild-type counterparts to challenges such as wounding or infection (33). Flaky tail, a spontaneously occurring mouse mutant with no genetically cleaved filaggrin protein, biochemically and histologically strongly resembles human ichthyosis vulgaris $(30,34)$. Again, these mice will be very useful for studying at the cellular level how keratinocytes compensate for the loss of filaggrin, a major structural protein. However, this model also raises the central question of how to interpret the presentation of human ichthyosis vulgaris or any other adult skin disorder on a haired animal. These experiments suggest that the need to protect the body from desiccation and infection demands redundancy at the protein and system-wide levels.

In addition to the mutant mouse strains created as animal models for specific human disorders, there is a growing list of mutant mouse strains with unexpected primary defects in epidermal barrier acquisition. For example, targeting of genes encoding Aryl hydrocarbon receptor nuclear translocator and Kelch-like ECHassociating protein 1 , transcription factors that modulate the response to hypoxia as well as oxidative and xenobiotic stress, respectively, demonstrate that these proteins have primary roles in establishment of the epidermal barrier $(35,36)$. The necessary role of these transcription factors in barrier acquisition raises the intriguing possibility that stress response signaling pathways may not only respond to barrier disruption, but may also have primary roles in barrier regulation. Additional studies have demonstrated novel functions for the transcription factors Klf4 and Grainyheadlike 3 in the proper establishment of the epidermal barrier $(15,37)$. The transcriptional regulation of proper epidermal differentiation was underscored by the manifestation of a barrier deficiency in mice with an epidermal-specific ablation of a component of the RNA polymerase II transcription preinitiation complex (38). Identification of the specific targets regulated by these transcription factors should aid in the elucidation of the hierarchy of pathways regulating barrier acquisition. Analysis of mice with other genetic ablations have elucidated additional proteins necessary for establishment of the barrier, such as membrane-anchored channelactivating serine protease 1 , serine protease inhibitor matriptase, 
tight junction protein claudin-1, and polarity protein E-cadherin $(7,39-41)$. Mice with mutations in regulatory molecules such as p63 or IKK $\alpha$ will not be discussed here since their primary defects are related to events earlier in the epidermal specification and differentiation processes, respectively. Along with biochemical studies, analysis of the transcriptional, protein, and lipid profiles of this array of mutant mouse models of disease will start to establish the hierarchy and branches of the signaling pathways required to establish the barrier and compensate for its loss. With these animal models, the analysis of multiple genetically identical newborns greatly enhances the elucidation of the barrier-specific defect. An initial clinical investigation of children diagnosed with ichthyosis and growth failure demonstrated a correlation between increased transepidermal water loss and caloric drain through heat evaporation (42). It is interesting to speculate that some human newborns that fail to thrive and lose weight (i.e., water) suffer from rare, undetected barrier disorders.

\section{Impaired barrier and skin inflammatory disorders: atopic dermatitis and psoriasis}

The barrier is established during in utero development, maintained throughout life, and reestablished after a breach. The study of congenital epidermal barrier disorders will help to elucidate the pathways necessary to establish the barrier and also hints at the existence of a complex molecular redundancy that is evoked to repair a disrupted barrier. Although these severe congenital defects are rare, impaired epidermal barrier function is a hallmark feature of 2 of the most common inflammatory skin disorders, psoriasis and $\mathrm{AD}(43-46)$. Psoriasis is a chronic skin disease in which very distinct erythrosquamous silvery scales cover either areas of inflection, the trunk and proximal extremities, or the entire body. Psoriasis affects $2-3 \%$ of the population, with typical age of onset in the third decade (46). As discussed below, the impaired barrier of psoriatic lesions may contribute to keratinocyte hyperproliferation and cytokine activation of lymphocytes. AD is a chronic inflammatory skin disease characterized by pruritus, eczema, and cutaneous hyperreactivity to environmental triggers (45). The onset of AD is typically within the first 6 months of life, and the disease currently affects $10-20 \%$ of children in developed countries. This prevalence has risen steadily in the last 30 years, suggesting that environmental factors contribute to pathogenesis (47). The impaired skin barrier function of $\mathrm{AD}$ patients may contribute to increased antigen absorption and result in cutaneous hyperreactivity (48). For both of these inflammatory skin disorders, the degree of epidermal barrier disruption correlates with severity of presentation, as measured by degree of inflammation or state of dermatitis $(45,48)$. However, these disorders are markedly different in skin presentation, gene expression changes, and immune infiltration.

Psoriasis and $\mathrm{AD}$ have both genetic and environmental components underlying their pathogenesis. One of the most intriguing findings from the genome-wide screens of $\mathrm{AD}$ and psoriasis susceptibility is that multiple loci are coincident. Genetic linkage of both $\mathrm{AD}$ and psoriasis susceptibility to the epidermal differentiation complex on chromosome 1q21, a 1.6-Mb locus of at least 30 genes encoding proteins that both build and regulate barrier formation, strongly suggests a role for barrier function or repair in these inflammatory disorders $(49,50)$. Resident within this epidermal differentiation complex on 1q21 are many excellent candidate genes that may initiate or maintain a skin inflammatory disorder. The clustering of these epidermal-specific genes raises the question of whether they are held together as a result of recent evolution through gene duplication or to enable coordinate gene regulation, as observed in the Globin or Hox gene clusters. A priori, the linkage of both $\mathrm{AD}$ and psoriasis susceptibility to the epidermal differentiation complex on chromosome 1q21 may be one of the most difficult to unravel, considering the possibility that more than one of the genes mapping to this region play a significant role in the progression of a skin inflammatory disorder.

Very recently, McLean and colleagues convincingly demonstrated that an impaired barrier contributes to $\mathrm{AD}$ with the discovery that common mutations in the gene encoding filaggrin, resident in the epidermal differentiation complex, are a strong predisposing factor for AD (51). Recalling classic studies that AD is particularly common in individuals with severe ichthyosis vulgaris, these scientists determined that $\mathrm{AD}$ is inherited as a semidominant trait in the pedigrees they analyzed and thus demonstrated that filaggrin deficiency underlies ichthyosis vulgaris. Recognizing that filaggrin mutations would be in linkage disequilibrium with other genes in the epidermal differentiation complex, they extended the AD studies to 3 additional populations. Notably, in all studies, mutations in filaggrin - in both homozygous and heterozygous states - were highly overrepresented in pediatric AD patients.

Interestingly, 2 of these large $\mathrm{AD}$ cohorts were ascertained on the basis of asthma. Approximately half of severe AD patients will develop asthma, and two-thirds will develop allergic rhinitis (also known as hay fever) (52). AD, asthma, and hay fever are epithelial organ-specific allergen responses of the skin, lung, and nose, respectively. The high concordance rate among the atopic disorders raises the question of whether there are shared genetic risks for all 3 disorders or whether antigen exposure in $\mathrm{AD}$ is the initiating event. In these recent cohort studies, mutations in filaggrin are selectively associated with individuals that have both asthma and $\mathrm{AD}$, with no association observed with asthma in the absence of $\mathrm{AD}$ (51). This finding has important clinical implications for medical management of children with $\mathrm{AD}$, since $\mathrm{AD}$ is often the earliest sign of the progression to asthma and hay fever known as the atopic march (52). A classic study in mice demonstrated that epicutaneous sensitization to an allergen can induce dermatitis and augment airway hyperreactivity, characteristics of AD and asthma, respectively (53). Both these animal and human cohort studies suggest that the increased allergen exposure through the skin of the AD infant may itself predispose to asthma. This hypothesis would argue for aggressive means to control the infant's AD before asthma develops. However, if impaired barrier recovery even in the seemingly unaffected areas underlies the genetic susceptibility to $\mathrm{AD}$ and contributes to the development of asthma, then controlling the outbreaks of AD lesions may not be sufficient to curtail the onset of asthma. Teasing apart the complex interaction of environmental challenges to the immunologic and epithelial systems in atopic disorders is a major direction of future research. The incomplete penetrance of heterozygous mutations in filaggrin resulting in either ichthyosis vulgaris or AD may be modified by the variant expression of other epidermal cornification genes also resident in the epidermal differentiation complex $(30,51)$.

In addition to these recent findings of filaggrin mutations underlying AD, earlier studies showed that a common E420K polymorphism in the lymphoepithelial SPINK5 protein (also known as LEKTI), encoded by the SPINK5 gene, contributes to the risk of developing AD (54). SPINK5, expressed in the outer layers of the epidermis, was discussed previously in this Review 
as the genetic defect underlying Netherton syndrome, which is characterized by congenital ichthyosis and AD (28). The Spink5deficient animal model may help to unravel whether inadequate barrier development or repair is an initiating event in AD. Initial studies show that transplantation of skin from Spink $5^{-/-}$mice to an immunocompromised mouse elicits an inflammatory infiltration in the dermis (55). Future studies involving allogenic grafting of Spink $5^{-/-}$murine skin to an immunocompetent mouse will reveal the full extent of the immune and epidermal response to Spink5 deficiency. Although some human disorders can be modeled with null alleles in the mouse, the more complex analysis of these multigenic traits will require the creation of hypomorphic alleles. In theory, a more sophisticated animal model of AD would be a mouse with a $\mathrm{E} 420 \mathrm{~K}$ amino acid change in the Spink5 protein. Unfortunately, the glutamic acid residue at amino acid 420 is not conserved in the mouse Spink5 protein, raising the question of how best to model the common role of SPINK5 in the predisposition to AD. Therefore, future studies need to be directed toward understanding the role of SPINK5 in epidermal differentiation and barrier function. As well, expression of SPINK5 in other epithelial and immune cells needs to be explored in both humans and mouse models to unravel whether the atopic disorders observed in these patients is intrinsic to defects in immune cell activation or in epithelial barriers. Although the most widely recognized feature of the atopic disorders is a misregulation of the immune system, these findings suggest that there may be ways in which an epithelial barrier is built or responds to stress that predispose to these disorders (56).

The genetic linkage of psoriasis to the epidermal differentiation complex has not yet been untangled and may similarly reflect reduced expression of 1 or more genes resident in the locus. Mice with an epidermal-specific deletion of the dimeric transcription factor complex activator protein 1 (AP1), consisting of c-Jun and JunB, demonstrate many of the histological and molecular hallmarks of psoriasis (57). Prior to any skin alterations in these mice, the chemotactic proteins S100A8 and S100A9 - which map to chromosome 1q21 - were found to be highly upregulated, as has also been reported in human psoriatic samples $(57,58)$. Located immediately proximal to S100A9 are 2 genes encoding peptidoglycan recognition proteins that have also recently been put forth as candidate modifiers of psoriasis (59). Also resident within the epidermal differentiation complex is a family of 10 closely related genes that encode small proline-rich proteins (SPRRs). SPRRs are cross-linked by TGM1 to establish the CE scaffold onto which other proteins are then subsequently cross-linked. SPRR genes are highly upregulated in both human psoriatic plaques and the skin of barrier-deficient mouse models $(33,60,61)$. Intriguingly, this upregulation of the SPRR genes is observed as a stress response in a variety of other tissues. Sprr genes are upregulated in bronchial epithelial and mononuclear cells after allergen challenge in an animal model of asthma (62). Similarly, Sprr genes are strongly upregulated in intestinal epithelium both in response to bacterial colonization and with induction of allergic gastrointestinal inflammation $(62,63)$. Duct ligation results in SPRR gene induction in biliary epithelial cells related to recovery of barrier function (64). These results suggest that there is a common stress response within epidermal, intestinal, bronchial, and biliary epithelium in response to infection. Are the SPRR proteins mediating a restoration of the barrier in multiple epithelial tissues? Alternatively, have the SPRR proteins been subverted as substrates for the CE but play an initial role in a primitive innate immune response? Interestingly, SPRR gene induction in cardiomyocytes responding to either biomechanical or ischemic stress renders a cardioprotective effect (65). This diverse expression data suggests epithelial barriers evoke a common stress response and that inappropriate recovery may contribute to the pathogenesis of inflammatory disorders.

One of the most intriguing findings from the genome-wide screens of $\mathrm{AD}$ and psoriasis susceptibility was that multiple identified regions, in addition to the epidermal differentiation complex, are coincident (50). One intriguing hypothesis to explain these common risks to develop either psoriasis or $\mathrm{AD}$ is that the underlying genes modulate how the skin repairs a breached barrier and whether the skin mounts an immune response. Breaches of the barrier are common events in our daily lives. For example, topical exposure to organic solvents or detergents that remove lipids from the stratum corneum cause a localized disruption. Scratching or mechanical stress that removes the upper layers of the skin also results in a local breach of this barrier. Using animal models and studies in patients, Elias, Feingold, and colleagues have demonstrated that after an initial rapid repair of this barrier by the lipids released from preformed granules, a slower recovery phase with de novo lipid synthesis follows (66). Two of the most striking results of barrier perturbation are the effects on DNA synthesis and cytokine production (67-69). Specifically, increased DNA synthesis is proportional to the degree of barrier perturbation and is partially restored to normal levels by occlusion of the skin with a water-impermeable membrane. Barrier disruption stimulates immediate production of cytokines, including TNF- $\alpha$, IFN- $\gamma$, IL- 1 , and GM-CSF $(67,69)$. This cytokine release acts in an autocrine fashion to induce differentiation and growth of keratinocytes and also functions in a paracrine and endocrine fashion to stimulate both local and systemic inflammatory and immune responses. These studies demonstrate that epidermal hyperplasia and cytokine induction are intrinsic epidermal responses to barrier disruption $(67,69,70)$.

Although aberrant lymphocyte activation is considered the root cause of skin inflammatory disorders, the discovery that barrier disruption initiates keratinocyte hyperproliferation and cytokine release suggested that there may be a feedback loop whereby the breach of barrier contributes to the activation of immune cells (69). Recent reports of keratinocyte-specific genetic alterations in animal models for psoriasis, including loss of the dimeric transcription factor complex AP1, consisting of c-Jun and JunB, or ectopic expression of activated STAT3 or TGF- $\beta$, expand the links between the keratinocyte and immunologic causes of this complex disorder $(57,71,72)$. Of note, these results could have direct translational importance, as activated STAT3 is expressed in the nuclei of human psoriatic lesions (72). As well, JunB maps to chromosome $19 \mathrm{p} 13,1$ of the 6 different psoriasis-susceptibility loci, and has reduced expression in the epidermis of psoriatic plaques (57). It will be interesting to determine whether these animal models have impaired barrier recovery as a component of the psoriatic progress.

To directly test the role of barrier restoration in inflammatory skin disorders, we compared the expression profile of genes misregulated in both barrier-deficient $\mathrm{Klf4}^{-{ }^{--}}$mouse skin and human psoriatic plaques $(15,73,74)$. Many similarities were identified, including an increased expression of gap junction proteins. Intriguingly, dominant-acting missense mutations in gap junction proteins underlie several rare ichthyoses including erythro- 


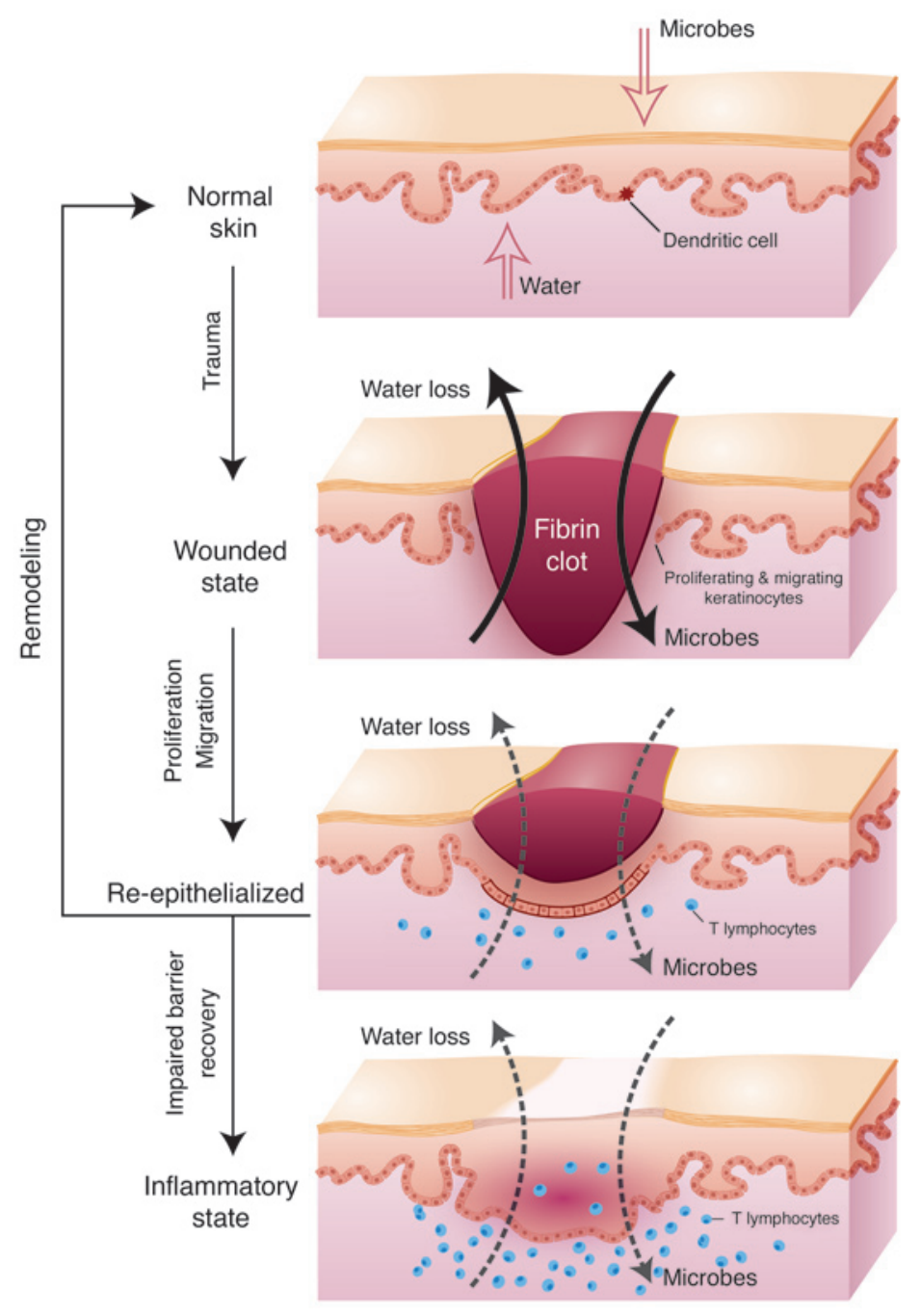

Figure 2

Role of barrier acquisition in the epidermal response to wound healing. Under normal conditions the epidermis serves as a barrier to retain water within the body and prevent the entry of infectious agents (e.g., microbes) or chemical agents. Dendritic Langerhans cells, resident in the epidermis, recognize, process, and present antigens to $T$ lymphocytes. In response to trauma to the epidermis, depicted as a full-thickness epidermal wound, keratinocytes increase their proliferation rate and cytokine release. Keratinocytes proliferate and migrate to re-epithelialize the wounded area. T lymphocytes are recruited into the damaged skin. As part of the normal process, keratinocytes initiate the process of terminal differentiation to restore the epidermal barrier. However, if the process of terminal differentiation or barrier recovery is impaired, the skin can enter an inflammatory state.

Even if impaired barrier recovery contributes to psoriasis and $\mathrm{AD}$, there must be additional factors that determine the type of immune response elicited since these disorders are characterized by distinct Th1/Th2 lymphocyte profiles. Beyond identifying the causative polymorphisms that underlie the common susceptibility to both AD and psoriasis, we will need to understand the complexity of how epidermal and immune cells interact to elicit both the keratinocyteand the T cell-specific manifestations of $\mathrm{AD}$ and psoriasis. One obvious possibility is that additional genetic or environmental risk factors modulate and specify the immune response. Much research focuses on the distinct Th1/Th2 lymphocyte infiltrations into psoriatic and AD skin.

The epidermal permeability barrier also has 2 distinct functions: retaining the water within the body and preventing the entry of dangerous substances. An animal model with an epidermal-specific targeted ablation of the adhesion molecule E-cadherin has a selective defect in water loss without any demonstrable defect in the entry of small molecules (41). These E-cadherin-deficient newborn mice have an increased

keratodermia variabilis, keratitis-ichthyosis-deafness syndrome, and Vohwinkel syndrome (75). Mimicking only the increased gap junctional communication observed in both $\mathrm{Klf4}^{-/-}$mice skin and human psoriatic plaques in transgenic mice resulted in congenital barrier deficiency (76). As described previously, the barrier is established in utero, maintained throughout life, and reestablished after trauma such as abrasion or wounding. Even when the epidermis has fully re-epithelialized, the ensuing scar at the site of trauma continues to exhibit mild barrier deficiency compared with uninvolved skin for as long as 1 year (77). Wounding is an initiating event in a significant percentage of psoriatic patients, which is clinically referred to as the isomorphic phenomenon. Interestingly, wounds on the mice with the increased gap junctional communication re-epithelialized at a normal rate. However, the inability to restore the barrier arrested the wounds in the hyperproliferative state and resulted in lymphocyte infiltration (76). These studies suggest that the signal to restore the homeostatic balance between proliferation and differentiation after re-epithelialization is restoration of the barrier and that in its absence the skin enters a pathologic state (Figure 2). Untangling some of these complex pathways will be a major area of future research, requiring the examination of tissue-specific genetic changes in both animal models and human patient samples. rate of transepidermal water loss, but they demonstrate a normal ability to exclude the penetration of small molecules through their skin. As described above, many of these animal models of congenital barrier deficiencies die perinatally. Future studies of the immune response to a barrier deficiency evoked in adult skin will require either allogenic grafts or postnatally induced gene ablation.

Still, some hints about the type of immune stimulation that might result from the impaired barrier in these genetically distinct animal models can be gleaned from examining their neonatal skin transcriptional profile. For example, barrier-deficient $\mathrm{Klf4}^{-/-}$newborn mice already exhibit high levels of the mRNA encoding the cytokine thymic stromal lymphopoietin (Tslp), which activates dendritic cells and is highly upregulated in the epithelial cells of involved skin from patients with $\mathrm{AD}$ as well as in bronchial epithelium of asthmatics $(78,79)$. Recent experiments in animal models have shown that transgenic expression of Tslp in skin and lung epithelium is sufficient to induce $\mathrm{AD}$ and allergic airway inflammation, respectively $(80,81)$. Moreover, TSLP receptor knockout mice do not develop an inflammatory response in the lung to inhaled antigen (82). The high level of expression of TSLP in keratinocytes of AD patients and barrier-deficient mouse skin predicts that the keratinocytes signal to the immune cells to aid in combating the influx of invasive pathogens. This model also predicts that a failure to repair the breach in 
the barrier would continue to activate the local immune response. Consistent with the role of TSLP in immune cell activation is a possible direct role for this cytokine in epidermal differentiation and barrier acquisition. Altogether, along with understanding the distinct immune cell responses of psoriasis and $\mathrm{AD}$, we need to investigate more specifically the nature of the epidermal barrier deficiency associated with these skin inflammatory disorders.

\section{Future research directions: genomic analysis of the barrier breach stress response}

The manifestation of a congenital ichthyosis disorder, transitioning from the collodion membrane to the hyperkeratotic plaques, suggests that barrier-deficient skin adapts to the terrestrial environment. Molecular experiments discussed in this Review suggest that the skin senses the barrier loss and induces a stress response to repair this breach. The discovery of antibiotics and the use of specialized emollients in the last 100 years have significantly altered our ability as a species to cope with a breach in barrier. However, one century is insignificant in evolutionary time. Our genomes have not adapted to the lower risk posed by a breach of barrier and continue to respond severely. We may be better served to dampen the body's inherent heightened stress response or to develop therapies selectively targeted against the strong stress response in order to improve quality of life. We may already be affecting barrier acquisition with standard treatment of inflammatory skin disorders that often includes the use of corticosteroids and emollients. As described above, corticosteroids not only act as immune suppressants but also accelerate epidermal barrier acquisition in utero $(12,13)$. However, corticosteroids elicit a complex response from keratinocytes and barrier function when administered shortterm versus long-term and in high versus low doses (83). Emollients serve not only to provide a moist environment for wound healing, but also decrease the "sensing" of barrier perturbation. Either dampening the epidermal response to barrier disruption or utilizing drug therapies that enhance barrier recovery may ameliorate some epithelial disorders that are more commonly perceived as inflammatory disorders.

Elucidation of the multiple ways in which a keratinocyte signals a barrier disruption to other cell types and maintains a stress response is a daunting task. Although a keratinocyte's initial response to a stressor may not occur at a transcriptional level, the changes necessary to maintain a stress response will be reflected in gene expression changes. Future experiments should define the expression profile changes that accompany a keratinocyte's response to individual stresses that are often sensed simultaneously, such as microbe infection, hypoxia, water loss, or oxidative stress. It will be equally instructive to monitor how the keratinocyte responds when restored to normal homeostatic conditions and "turns off" the stress response. Existing algorithms can be utilized to determine both specific and common transcriptional responses elicited by stresses. Although this is an ambitious project, this type of model has been assembled to examine how the yeast Saccharomyces cerevisiae responds to changes in environment, food availability, and developmental state (84).

To gain a more comprehensive understanding of the stress response, investigators must elucidate further how these changes are regulated at the transcription level. Toward this end, future experiments need to identify both the transcription factors and the DNA sequences to which they bind that regulate a stress response. Transcriptional profiling of keratinocytes deficient in a transcription factor essential to epidermal differentiation under stressed conditions may establish the hierarchy of some regulated pathways. RNA interference, a novel technique that interferes with transcription of a specific RNA in mammalian cells, can be used to deplete keratinocytes of a putative regulatory protein and thus determine a specific protein's necessary role in maintaining the stress response (85).

Although this is also a challenging project, novel technologies are being developed as part of the Human Genome Project's goal to identify all functional elements in the human genome sequence (86). Analysis of the complete sequence of human, mouse, chimpanzee, and dog genomes has revealed that approximately $5 \%$ of our DNA is under strong positive selection (87). Approximately half of this conserved sequence encodes proteins or RNA genes. The function of the other half, the conserved noncoding sequences, is hypothesized to regulate the complex expression of nearby genes (86).

Multiple examples have been published demonstrating the role of conserved noncoding sequences resident in the proximal promoter or intron sequences of a gene that regulate its temporal or spatial expression. One intriguing example of this is the identification of a single nucleotide polymorphism in a runt-related transcription factor 1 (RUNX1) binding site that is associated with susceptibility to psoriasis (88). Some conserved noncoding sequences exert long-range effects on gene transcription, such as the locus control region regulating the developmentally regulated switching in the $\beta$-globin gene complex (89). Another example of a conserved noncoding sequence is a long-range enhancer of the Sprr genes that has no activity during normal homeostatic growth of keratinocytes but serves as a strong enhancer when the stress response is activated to upregulate Sprr gene expression (90). It is intriguing to speculate that polymorphisms in the regulatory sequences that modulate the barrier recovery response may determine the susceptibility to $\mathrm{AD}$ and psoriasis. Perhaps a limited amount of the conserved noncoding sequence in our genomes regulates gene expression under homeostatic conditions, and a significant proportion encodes the complex stress response. Keratinocytes can be grown as monolayer or organotypic cultures or grafted as full-thickness skin onto mice, making this one of the most tractable systems to investigate complex gene regulation. With further analysis, we can gain insight into a multitude of common and rare human skin disorders.

\section{Acknowledgments}

This work is supported by the National Human Genome Research Institute Intramural Program. Darryl Leja prepared the illustrations. I am deeply indebted to David Nathan, Leonard Milstone, Jonathan Vogel, Mark Udey, and Cristina Strong for critical evaluation and suggestions.

Address correspondence to: Julie A. Segre, Building 49, Room 4A26, 49 Convent Drive, MSC 4442, Bethesda, Maryland 208924442, USA. Phone: (301) 402-2314; Fax: (301) 402-4929; E-mail: jsegre@nhgri.nih.gov.
1. Ito, M., et al. 2005. Stem cells in the hair follicle bulge contribute to wound repair but not to homeostasis of the epidermis. Nat. Med. 11:1351-1354.

2. Fuchs, E., and Raghavan, S. 2002. Getting under the skin of epidermal morphogenesis. Nat. Rev. Genet. 3:199-209.

3. Niemann, C., and Watt, F.M. 2002. Designer skin: lineage commitment in postnatal epidermis. Trends
Cell Biol. 12:185-192.

4. Elias, P.M. 1983. Epidermal lipids, barrier function, and desquamation. J. Invest. Dermatol. 80:44s-49s.

5. Kalinin, A.E., Kajava, A.V., and Steinert, P.M. 2002. 
Epithelial barrier function: assembly and structural features of the cornified cell envelope. Bioessays. 24:789-800.

6. Nemes, Z., and Steinert, P.M. 1999. Bricks and mortar of the epidermal barrier. Exp. Mol. Med. 31:5-19.

7. Furuse, M., et al. 2002. Claudin-based tight junctions are crucial for the mammalian epidermal barrier: a lesson from claudin-1-deficient mice. J. Cell Biol. 156:1099-1111.

8. Steinert, P.M. 2000. The complexity and redundancy of epithelial barrier function. J. Cell Biol. 151:F5-F8.

9. Segre, J. 2003. Complex redundancy to build a simple epidermal permeability barrier. Curr. Opin. Cell Biol. 15:776-782.

10. Morita, K. et al. 2002. Molecular architecture of tight junctions of periderm differs from that of the maculae occludentes of epidermis. J. Invest. Derma tol. 118:1073-1079.

11. Kalia, Y.N., Nonato, L.B., Lund, C.H., and Guy, R.H. 1998. Development of skin barrier function in premature infants. J. Invest. Dermatol. 111:320-326.

12. Aszterbaum, M., Feingold, K.R., Menon, G.K., and Williams, M.L. 1993. Glucocorticoids accelerate fetal maturation of the epidermal permeability barrier in the rat. J. Clin. Invest. 91:2703-2708.

13. Hardman, M.J., Sisi, P., Banbury, D.N., and Byrne, C. 1998. Patterned acquisition of skin barrier function during development. Development. 125:1541-1552.

14. Hanley, K., et al. 1998. Glucocorticoid deficiency delays stratum corneum maturation in the fetal mouse. J. Invest. Dermatol. 111:440-444.

15. Segre, J.A., Bauer, C., and Fuchs, E. 1999. Klf4 is a transcription factor required for establishing the barrier function of the skin. Nat. Genet. 22:356-360.

16. Jaubert, J., Cheng, J., and Segre, J.A. 2003. Ectopic expression of kruppel like factor 4 (Klf4) accelerates formation of the epidermal permeability barrier. Development. 130:2767-2777.

17. Marshall, D., Hardman, M.J., Nield, K.M., and Byrne, C. 2001. Differentially expressed late constituents of the epidermal cornified envelope. Proc Natl. Acad. Sci. U. S. A. 98:13031-13036.

18. Richard, G. 2004. Molecular genetics of the ichthyoses. Am. J. Med. Genet. C Semin. Med. Genet. 131C:32-44.

19. Huber, M., et al. 1995. Mutations of keratinocyte transglutaminase in lamellar ichthyosis. Science. 267:525-528.

20. Russell, L.J., et al. 1995. Mutations in the gene for transglutaminase 1 in autosomal recessive lamellar ichthyosis. Nat. Genet. 9:279-283.

21. Lefevre, C., et al. 2003. Mutations in the transporter ABCA12 are associated with lamellar ichthyosis type 2. Hum. Mol. Genet. 12:2369-2378.

22. Lefevre, C., et al. 2006. Mutations in a new cytochrome P450 gene in lamellar ichthyosis type 3 Hum. Mol. Genet. 15:767-776.

23. Jobard, F., et al. 2002. Lipoxygenase-3 (ALOXE3) and 12(R)-lipoxygenase (ALOX12B) are mutated in non-bullous congenital ichthyosiform erythroderma (NCIE) linked to chromosome 17p13.1. Hum. Mol. Genet. 11:107-113.

24. Lefevre, C., et al. 2004. Mutations in ichthyin a new gene on chromosome $5 \mathrm{q} 33$ in a new form of autosomal recessive congenital ichthyosis. Hum. Mol. Genet. 13:2473-2482.

25. Williams, M.L., and Elias, P.M. 1985. Heterogeneity in autosomal recessive ichthyosis. Clinical and biochemical differentiation of lamellar ichthyosis and nonbullous congenital ichthyosiform erythroderma. Arch. Dermatol. 121:477-488.

26. Kelsell, D.P., et al. 2005. Mutations in ABCA12 underlie the severe congenital skin disease harlequin ichthyosis. Am. J. Hum. Genet. 76:794-803.

27. Akiyama, M., et al. 2005. Mutations in lipid transporter ABCA12 in harlequin ichthyosis and functional recovery by corrective gene transfer. J. Clin. Invest. 115:1777-1784. doi:10.1172/JCI24834.
28. Chavanas, S., et al. 2000. Mutations in SPINK5, encoding a serine protease inhibitor, cause Netherton syndrome. Nat. Genet. 25:141-142.

29. Raghunath, M., et al. 2003. Self-healing collodion baby: a dynamic phenotype explained by a particular transglutaminase-1 mutation. J. Invest. Dermatol. 120:224-228.

30. Smith, F.J., et al. 2006. Loss-of-function mutations in the gene encoding filaggrin cause ichthyosis vulgaris. Nat. Genet. 38:337-342.

31. Matsuki, M., et al. 1998. Defective stratum corneum and early neonatal death in mice lacking the gene for transglutaminase 1 (keratinocyte transglutaminase). Proc. Natl. Acad. Sci. U. S. A. 95:1044-1049.

32. Kuramoto, N., et al. 2002. Development of ichthyosiform skin compensates for defective permeability barrier function in mice lacking transglutaminase 1. J. Clin. Invest. 109:243-250. doi:10.1172/ JCI200213563.

33. Koch, P.J., et al. 2000. Lessons from loricrin-deficient mice: compensatory mechanisms maintaining skin barrier function in the absence of a major cornified envelope protein. J. Cell Biol. 151:389-400

34. Presland, R.B., et al. 2000. Loss of normal profilaggrin and filaggrin in flaky tail (ft/ft) mice: an animal model for the filaggrin-deficient skin disease ichthyosis vulgaris. J. Invest. Dermatol. 115:1072-1081.

35. Wakabayashi, N., et al. 2003. Keap1-null mutation leads to postnatal lethality due to constitutive Nrf2 activation. Nat. Genet. 35:238-245.

36. Takagi, S., et al. 2003. Alteration of the 4-sphingenine scaffolds of ceramides in keratinocytespecific Arnt-deficient mice affects skin barrier function. J. Clin. Invest. 112:1372-1382. doi:10.1172/JCI200318513.

37. Ting, S.B., et al. 2005. A homolog of Drosophila grainy head is essential for epidermal integrity in mice. Science. 308:411-413.

38. Indra, A.K., et al. 2005. TAF10 is required for the establishment of skin barrier function in foetal, but not in adult mouse epidermis. Dev. Biol. 285:28-37.

39. Leyvraz, C., et al. 2005. The epidermal barrier function is dependent on the serine protease CAP1/ Prss8. J. Cell Biol. 170:487-496.

40. List, K., et al. 2002. Matriptase/MT-SP1 is required for postnatal survival, epidermal barrier function, hair follicle development, and thymic homeostasis. Oncogene. 21:3765-3779.

41. Tunggal, J.A., et al. 2005. E-cadherin is essential for in vivo epidermal barrier function by regulating tight junctions. EMBO J. 24:1146-1156.

42. Moskowitz, D.G., et al. 2004. Pathophysiologic basis for growth failure in children with ichthyosis: an evaluation of cutaneous ultrastructure, epidermal permeability barrier function, and energy expenditure. J. Pediatr. 145:82-92.

43. Esche, C., de Benedetto, A., and Beck, L.A. 2004. Keratinocytes in atopic dermatitis: inflammatory signals. Curr. Allergy Asthma Rep. 4:276-284.

44. Ghadially, R., Reed, J.T., and Elias, P.M. 1996. Stratum corneum structure and function correlates with phenotype in psoriasis. J. Invest. Dermatol. 107:558-564

45. Leung, D.Y., Boguniewicz, M., Howell, M.D., Nomura, I., and Hamid, Q.A. 2004. New insights into atopic dermatitis. J. Clin. Invest. 113:651-657. doi:10.1172/JCI200421060.

46. Nickoloff, B.J., and Nestle, F.O. 2004. Recent insights into the immunopathogenesis of psoriasis provide new therapeutic opportunities. J. Clin. Invest. 113:1664-1675. doi:10.1172/ JCI200422147.

47. Johansson, S.G., et al. 2001. A revised nomenclature for allergy. An EAACI position statement from the EAACI nomenclature task force. Allergy. 56:813-824

48. Sator, P.G., Schmidt, J.B., and Honigsmann, H.
2003. Comparison of epidermal hydration and skin surface lipids in healthy individuals and in patients with atopic dermatitis. J. Am. Acad. Dermatol. 48:352-358.

49. Capon, F., et al. 2001. Fine mapping of the PSORS4 psoriasis susceptibility region on chromosome 1q21. J. Invest. Dermatol. 116:728-730.

50. Cookson, W.O., et al. 2001. Genetic linkage of childhood atopic dermatitis to psoriasis susceptibility loci. Nat. Genet. 27:372-373.

51. Palmer, C.N.A., et al. 2006. Common loss-of-funtion variants of the epidermal barrier protein filaggrin are a major predisposing factor for atopic dermatitis. Nat. Genet. doi:10.1038/ng1767.

52. Spergel, J.M., and Paller, A.S. 2003. Atopic dermatitis and the atopic march. J. Allergy Clin. Immunol. 112:S118-S127.

53. Spergel, J.M., et al. 1998. Epicutaneous sensitization with protein antigen induces localized allergic dermatitis and hyperresponsiveness to methacholine after single exposure to aerosolized antigen in mice. J. Clin. Invest. 101:1614-1622.

54. Walley, A.J., et al. 2001. Gene polymorphism in Netherton and common atopic disease. Nat. Genet. 29:175-178.

55. Descargues, P., et al. 2005. Spink5-deficient mice mimic Netherton syndrome through degradation of desmoglein 1 by epidermal protease hyperactivity. Nat. Genet. 37:56-65.

56. Cookson, W. 2004. The immunogenetics of asthma and eczema: a new focus on the epithelium. Nat. Rev. Immunol. 4:978-988.

57. Zenz, R., et al. 2005. Psoriasis-like skin disease and arthritis caused by inducible epidermal deletion of Jun proteins. Nature. 437:369-375.

58. Broome, A.M., Ryan, D., and Eckert, R.L. 2003. S100 protein subcellular localization during epidermal differentiation and psoriasis. J. Histochem. Cytochem. 51:675-685.

59. Sun, C., et al. 2006. Peptidoglycan recognition proteins Pglyrp3 and Pglyrp4 are encoded from the epidermal differentiation complex and are candidate genes for the Psors4 locus on chromosome 1q21. Hum. Genet. 119:113-125.

60. Patel, S., Kartasova, T., and Segre, J.A. 2003. Mouse Sprr locus: a tandem array of coordinately regulated genes. Mamm. Genome. 14:140-148.

61. Zhou, X., et al. 2003. Novel mechanisms of T-cell and dendritic cell activation revealed by profiling of psoriasis on the 63,100-element oligonucleotide array. Physiol. Genomics. 13:69-78.

62. Zimmermann, N., et al. 2005. Expression and regulation of small proline-rich protein 2 in allergic inflammation. Am. J. Respir. Cell Mol. Biol. 32:428-435.

63. Hooper, L.V., et al. 2001. Molecular analysis of commensal host-microbial relationships in the intestine. Science. 291:881-884.

64. Nozaki, I., et al. 2005. Small proline-rich proteins 2 are noncoordinately upregulated by IL-6/STAT3 signaling after bile duct ligation. Lab. Invest. 85:109-123.

65. Pradervand, S., et al. 2004. Small proline-rich protein $1 \mathrm{~A}$ is a gp130 pathway- and stress-inducible cardioprotective protein. EMBOJ. 23:4517-4525.

66. Ghadially, R., Brown, B.E., Sequeira-Martin, S.M., Feingold, K.R., and Elias, P.M. 1995. The aged epidermal permeability barrier. Structural, functional, and lipid biochemical abnormalities in humans and a senescent murine model. J. Clin. Invest. 95:2281-2290

67. Nickoloff, B.J., and Naidu, Y. 1994. Perturbation of epidermal barrier function correlates with initiation of cytokine cascade in human skin. J. Am. Acad. Dermatol. 30:535-546.

68. Proksch, E., Feingold, K.R., Man, M.Q., and Elias, P.M. 1991. Barrier function regulates epidermal DNA synthesis. J. Clin. Invest. 87:1668-1673.

69. Wood, L.C., Jackson, S.M., Elias, P.M., Grunfeld, C., 
and Feingold, K.R. 1992. Cutaneous barrier perturbation stimulates cytokine production in the epidermis of mice. J. Clin. Invest. 90:482-487.

70. Wood, L.C., et al. 1996. Barrier disruption stimulates interleukin-1 alpha expression and release from a pre-formed pool in murine epidermis. J. Invest. Dermatol. 106:397-403.

71. Li, A.G., Wang, D., Feng, X.H., and Wang, X.J. 2004. Latent TGFbeta1 overexpression in keratinocytes results in a severe psoriasis-like skin disorder. EMBO J. 23:1770-1781.

72. Sano, S., et al. 2005. Stat3 links activated keratinocytes and immunocytes required for development of psoriasis in a novel transgenic mouse model. Nat. Med. 11:43-49.

73. Labarthe, M.P., Bosco, D., Saurat, J.H., Meda, P., and Salomon, D. 1998. Upregulation of connexin 26 between keratinocytes of psoriatic lesions. J. Invest. Dermatol. 111:72-76.

74. Lucke, T., et al. 1999. Upregulation of connexin 26 is a feature of keratinocyte differentiation in hyperproliferative epidermis, vaginal epithelium, and buccal epithelium. J. Invest. Dermatol. 112:354-361.

75. Richard, G. 2005. Connexin disorders of the skin. Clin. Dermatol. 23:23-32.

76. Djalilian, A.R., et al. 2006. Connexin 26 regulates epidermal barrier and wound remodeling and promotes psoriasiform response. J. Clin. Invest.
116:1243-1253. doi:10.1172/JCI27186.

77. Suetake, T., Sasai, S., Zhen, Y.X., Ohi, T., and Tagami, H. 1996. Functional analyses of the stratum corneum in scars. Sequential studies after injury and comparison among keloids, hypertrophic scars, and atrophic scars. Arch. Dermatol. 132:1453-1458.

78. Ying, S., et al. 2005. Thymic stromal lymphopoietin expression is increased in asthmatic airways and correlates with expression of Th2-attracting chemokines and disease severity. J. Immunol. 174:8183-8190.

79. Soumelis, V., et al. 2002. Human epithelial cells trigger dendritic cell mediated allergic inflammation by producing TSLP. Nat. Immunol. 3:673-680.

80. Zhou, B., et al. 2005. Thymic stromal lymphopoietin as a key initiator of allergic airway inflammation in mice. Nat. Immunol. 6:1047-1053.

81. Yoo, J., et al. 2005. Spontaneous atopic dermatitis in mice expressing an inducible thymic stromal lymphopoietin transgene specifically in the skin. J. Exp. Med. 202:541-549.

82. Al-Shami, A., Spolski, R., Kelly, J., Keane-Myers, A., and Leonard, W.J. 2005. A role for TSLP in the development of inflammation in an asthma model. J. Exp. Med. 202:829-839.

83. Kao, J.S., et al. 2003. Short-term glucocorticoid treatment compromises both permeability barrier homeostasis and stratum corneum integrity: inhibition of epidermal lipid synthesis accounts for functional abnormalities. J. Invest. Dermatol. 120:456-464

84. Lee, T.I., et al. 2002. Transcriptional regulatory networks in Saccharomyces cerevisiae. Science. 298:799-804.

85. Huppi, K., Martin, S.E., and Caplen, N.J. 2005 Defining and assaying RNAi in mammalian cells. Mol. Cell. 17:1-10.

86. ENCODE Project consortium. 2004. The ENCODE (ENCyclopedia Of DNA Elements) Project. Science. 306:636-640.

87. Lindblad-Toh, K., et al. 2005. Genome sequence, comparative analysis and haplotype structure of the domestic dog. Nature. 438:803-819.

88. Helms, C., et al. 2003. A putative RUNX1 binding site variant between SLC9A3R1 and NAT9 is associated with susceptibility to psoriasis. Nat. Genet. 35:349-356.

89. King, D.C., et al. 2005. Evaluation of regulatory potential and conservation scores for detecting cisregulatory modules in aligned mammalian genome sequences. Genome Res. 15:1051-1060.

90. Martin, N., Patel, S., and Segre, J.A. 2004. Longrange comparison of human and mouse Sprr loci to identify conserved noncoding sequences involved in coordinate regulation. Genome Res. 14:2430-2438. 